\title{
ArcheoSciences
}

Revue d'archéométrie

\section{Apport du lidar à la connaissance de l'histoire de l'occupation du sol en forêt de Haye}

Contribution of Lidar Data to the Study of Land-use History in Haye Forest

Murielle Georges-Leroy, Jérôme Bock, Étienne Dambrine et Jean-Luc Dupouey

\section{(C) OpenEdition}

12 Journals

\section{Édition électronique}

URL : https://journals.openedition.org/archeosciences/3015

DOI : $10.4000 /$ archeosciences.3015

ISBN : 978-2-7535-1849-0

ISSN : $2104-3728$

Éditeur

Presses universitaires de Rennes

Édition imprimée

Date de publication : 30 avril 2011

Pagination : 117-129

ISBN : 978-2-7535-1847-6

ISSN : $1960-1360$

\section{Référence électronique}

Murielle Georges-Leroy, Jérôme Bock, Étienne Dambrine et Jean-Luc Dupouey, « Apport du lidar à la connaissance de l'histoire de l'occupation du sol en forêt de Haye », ArcheoSciences [En ligne], 35 |

2011, mis en ligne le 30 avril 2013, consulté le 15 février 2023. URL : http://journals.openedition.org/ archeosciences/3015; DOI : https://doi.org/10.4000/archeosciences.3015

\section{cc)

Creative Commons - Attribution - Pas d'Utilisation Commerciale - Pas de Modification 4.0 International - CC BY-NC-ND 4.0

https://creativecommons.org/licenses/by-nc-nd/4.0/ 


\title{
Apport du lidar à la connaissance de l'histoire de l'occupation du sol en forêt de Haye
}

\section{Contribution of Lidar Data to the Study of Land-use History in Haye Forest}

\author{
Murielle Georges-Leroy*, Jérôme Bock**, Étienne Dambrine*** \\ et Jean-Luc DupoueY****
}

\begin{abstract}
Résumé : Le potentiel archéologique des forêts est fort, notamment en raison de la bonne conservation des vestiges sous forme de micro-reliefs, mais les prospections archéologiques sont gênées par le couvert forestier, ce qui freine l'étude des sites. Une méthode de télédétection assez récente, le scanneur laser aéroporté ou lidar, permet de s'affranchir d'une partie des contraintes physiques et permet le repérage et la cartographie des vestiges. Cette technique a été appliquée au massif forestier de Haye, qui couvre 116 km² à côté de Nancy, durant l'hiver 2006-2007. Elle a permis des apports considérables par rapport aux méthodes de prospection traditionnelles au sol jusque-là utilisées pour étudier les structures agraires et parcellaires et les habitats antiques qui y sont conservés. Ces apports ont porté sur de nombreux points : nette augmentation du nombre de vestiges découverts, amélioration de la localisation des vestiges initialement cartographiés au topofil et à la boussole ou au GPS, apports morphologiques et chronologiques, etc. Nous avons mis au point une méthode de cartographie et élaboré un référentiel de vestiges archéologiques et de structures morphologiques diverses, adaptés à l'analyse et à l'interprétation des données lidar. Au final, cette technique rend possible un véritable changement d'échelle dans l'étude des sites archéologiques et permet d'esquisser dans ses grandes lignes l'histoire de l'occupation du sol de l'actuel massif forestier de Haye.
\end{abstract}

\begin{abstract}
The archaeological potential of forests is high, mainly because of good conservation of the remains in the form of microreliefs, but the archaeological surveys are hampered by the forest cover, which slows the study of sites. A method of remote sensing, airborne laser scanning (lidar), overcomes some of the physical constraints and allows the identification and mapping of the remains. This technique was applied to Haye Forest, near Nancy, which covers $116 \mathrm{~km}^{2}$, in the winter of 2006-2007. Compared to traditional ground surveys, lidar improved substantially the study of ancient agrarian structures and settlements preserved in the forest. The mains inputs were: increase in the number of remains found, improved location of the remains originally mapped using topofil and compass or GPS, morphological and chronological informations, etc. We have developed a mapping method and a reference framework for archaeological remains and morphological structures, suitable for the analysis and interpretation of lidar data. Finally, this technique makes possible a real change of scale in the study of forest sites and allows to sketch in broad outline the land-use history of the Haye Forest.
\end{abstract}

Mots clé : forêt, lidar, parcellaire, prospection archéologique, scanneur laser aéroporté, structure agraire

Keywords : forest, lidar, field system, archaeological survey, airborne laser scanning, agrarian structure, land use

\footnotetext{
* DRAC de Lorraine, SRA - 6, place de Chambre, 57045 Metz cedex 1; UMR 6249 Chrono-Environnement. (murielle.leroy@culture.gouv.fr)

** ONF, Pôle R\&D, DT Lorraine - Bâtiment802-54840 Velaine-en-Haye. (jerome.bock@onf.fr)

*** INRA-Université de Savoie - 73376 Le-Bourget-du-Lac cedex. (etienne.dambrine@univ.savoie.fr)

**** INRA-Université de Lorraine, UMR 1137 Écologie et Écophysiologie forestières - 54280 Seichamps. (dupouey@nancy.inra.fr)
} 


\section{INTRODUCTION}

La forêt constitue un milieu avec un potentiel archéologique très fort, notamment du fait de la bonne conservation des vestiges sous forme de micro-reliefs, mais aussi par la mémoire que les sols forestiers gardent de ces occupations anciennes. Ainsi, les grands massifs forestiers situés sur les plateaux calcaires du Bajocien entre Pont-à-Mousson et Neufchâteau (Meurthe-et-Moselle et Vosges) recèlent-ils de nombreux habitats et parcellaires fossiles gallo-romains, objets d'un programme de recherche pluridisciplinaire depuis 1998 (Georges-Leroy et al., 2003 ; 2007). Ceux-ci se présentent sous la forme de pierriers linéaires (murées) ou de talus, parfois longs de plusieurs centaines de mètres et hauts en général de quelques dizaines de centimètres, qui délimitent des anciens champs ou pâtures, des enclos, mais aussi des voies, et sont associés à des habitats gallo-romains également conservés sous forme de micro-reliefs. Ces occupations anciennes ont encore un impact fort sur la biodiversité et la fertilité forestière actuelles, comme l'ont montré plusieurs études (Dupouey et al., 2002; 2007).

La forêt représente toutefois un milieu contraignant pour les prospections archéologiques : visibilité limitée à cause de la végétation, voire inaccessibilité à certains secteurs, difficulté (voire impossibilité) à repérer les vestiges totalement enfouis, difficulté à cartographier les sites, etc. (GeorgesLeroy et al., 2010). Ces contraintes, mais aussi le fréquent postulat d'un territoire forestier se rétrécissant progressivement depuis les défrichements néolithiques, ce qui implique l'existence de noyaux forestiers vierges de toute occupation agricole importante, expliquent l'absence de prospections archéologiques dans de nombreuses forêts et en conséquence le faible nombre de sites identifiés.

Une méthode assez récente de télédétection permet de s'affranchir d'une partie de ces contraintes et limites et peut aider au repérage et à la cartographie des vestiges archéologiques. Il s'agit du scanneur laser aéroporté, également connu sous l'acronyme anglais de lidar (light detection and ranging). Cette technique de télédétection a été utilisée pour la première fois en milieu forestier, à des fins archéologiques, à partir de 2003, par une équipe de l'institut de gestion des paysages de l'université de Fribourg-en-Brisgau sur des champs bombés conservés en forêt à Rastatt, en pays de Bade (Sittler, 2007). Des massifs forestiers ont ainsi été explorés dans divers pays, comme par exemple la forêt de Dean en Grande-Bretagne (Devereux et al., 2005), celle de la montagne de la Leitha en Autriche (Doneus et al., 2008), à Elverum en Norvège (Risbøl et al., 2006) ou dans le Michigan aux États-Unis (Gallagher et Josephs, 2008). Une expérience s'est aussi révélée fructueuse dans la forêt tropicale en Guyane française (Mestre et al., 2008). Les parcellaires et les vestiges agraires linéaires sont très bien révélés par lidar. Ce fait a été mis en évidence dès les premières utilisations de la méthode en 2000 (Motkin, 2001). En plus des champs bombés de Rastatt, cette méthode a notamment servi à la détection de Celtic fields aux Pays-Bas (Humme et al., 2006; Kooistra et Maas, 2008), de parcellaires autour du site de Stonehenge (Bewley, 2005) et dans la forêt de Dean, où ont aussi été repérés des rideaux de culture (Devereux et al., 2005).

Cette technique de télédétection a été utilisée pour la première fois en France, en contexte archéologique, durant l'hiver 2006-2007, avec notamment le vol au-dessus des 11600 ha du massif forestier de Haye, situé à côté de Nancy (fig. 1). Étudié dans le cadre du programme de recherche sur les parcellaires fossiles du plateau bajocien (Georges-Leroy $e t$ al., sous presse), ce massif forestier, essentiellement constitué de feuillus (hêtraie-chênaie-charmaie principalement), se localise dans une boucle de la Moselle entre Nancy et Toul, en limite sud de la confluence avec la Meurthe. En raison de sa position sur le plateau en bordure de la cuesta des Côtes de Moselle, il est entaillé par de profonds vallons et limité au nord, à l'est et au sud par de fortes côtes. Il a été très durement touché par la tempête du 26 décembre 1999, rendant

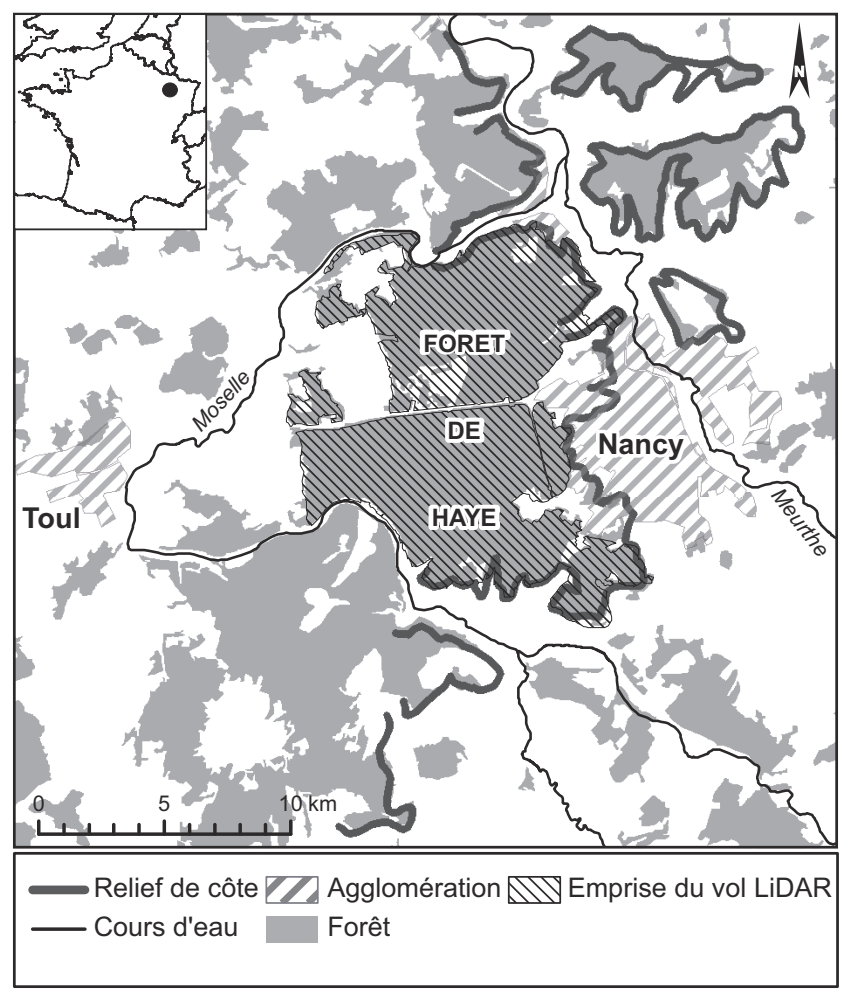

Figure 1 : Le massif forestier de Haye.

Figure 1: Haye Forest. 
certains secteurs inaccessibles aux prospections au sol pour plusieurs années (présence de ronces, régénérations denses, chablis, etc.). Les vestiges antiques conservés dans cette forêt ont fait l'objet de relevés et de sondages en quelques points du massif, dans les années 1970, par deux archéologues bénévoles, Michel Loiseau et Étienne Louis. À partir de 1995, une cartographie assez systématique des parcellaires a ensuite été entreprise par Philippe Loué, agent de l'Office National des Forêts (ONF). Notre équipe a poursuivi ces levés à partir de 1999 à l'aide de GPS à correction différentielle, les levés précédents ayant été réalisés au décamètre ou au topofil et à la boussole. L'erreur de positionnement a varié de quelques mètres à quelques centaines de mètres selon les modes d'acquisition et les zones prospectées. Cette imprécision sur les secteurs déjà cartographiés et l'importance des secteurs restant encore à cartographier (plusieurs milliers d'hectares à la fin 2005), dont certains inaccessibles au sol, ont conduit l'équipe à se tourner vers le scanneur laser aéroporté.

Le premier objectif de cette étude par télédétection était donc la réalisation de la cartographie la plus complète et la plus précise possible des vestiges de parcellaires et d'habitats anciens. Mais elle était aussi destinée à acquérir des informations sur les peuplements forestiers, afin notamment de mesurer la hauteur des arbres, car, en foresterie, la hauteur à un âge donné est un indicateur de la fertilité des sols (Pardé et Bouchon, 1988). L'objectif final est le croisement des données archéologiques et écologiques, afin d'analyser l'impact de l'utilisation ancienne des sols sur l'état et le fonctionnement des écosystèmes forestiers actuels (diversité végétale, productivité des peuplements).

Cet article vise à :

- décrire les étapes du protocole d'acquisition et, surtout, d'analyse des données récoltées afin d'aider à la réalisation de projets ultérieurs;

- présenter les apports de cette technique à l'étude des vestiges archéologiques conservés sous couvert forestier, notamment par rapport aux méthodes traditionnelles de prospection au sol, en s'appuyant sur l'exemple des habitats et des structures agraires et parcellaires du massif forestier de Haye.

\section{LA TÉLÉDÉTECTION \\ PAR SCANNEUR LASER AÉROPORTÉ}

\section{Principes généraux}

Le principe du levé lidar consiste en un survol à basse altitude de la zone d'étude à l'aide d'un avion équipé d'une source laser qui balaye très rapidement la surface terrestre, par bandes de quelques centaines de mètres de largeur (Joinville et al., 2003; Bilodeau et Deroin, 2008). Les impulsions laser sont interceptées par le sol ou par les objets en sursol (végétation, bâtiments) et réfléchies en direction de l'avion où un capteur détecte l'intensité et le temps de retour du signal. Ce temps de retour dépend de l'altitude du point d'impact qui est ainsi calculée. Des systèmes de positionnement dans l'avion (GPS, centrale inertielle) et au sol (GPS), permettent de recalculer la trajectoire de l'avion et d'en déduire la position des points d'impact avec une précision de quelques centimètres en altimétrie et de quelques dizaines de centimètres en planimétrie.

Comme une même impulsion lumineuse peut toucher à la fois la végétation (branches, troncs, feuilles, etc.) et le sol, les scanneurs enregistrent, selon les modèles, de deux à cinq échos (système multi-échos, utilisé en forêt de Haye). Il existe également depuis 2004 des systèmes qui enregistrent le signal de retour en continu (système full-waveform; Mallet et Bretar, 2009). Dans les deux types de système, il en résulte un nuage de points en trois dimensions. Les derniers échos renvoyés par chaque impulsion font ensuite l'objet d'une classification à l'aide d'algorithmes de filtrage permettant de séparer les points ayant atteint le sol de ceux ayant touché la végétation ou d'autres objets (points dits de «sursol») ${ }^{1}$.

Plusieurs modèles numériques d'élévation (MNE) peuvent être élaborés avec ces points, le plus souvent sous la forme d'une grille régulière d'altitudes dont la finesse de résolution dépend de la densité de points disponibles. Les points sol permettent de construire un modèle numérique de terrain (MNT) et les premiers échos de chaque impulsion permettent de réaliser un modèle numérique de surface (MNS), qui représente la surface terrestre avec les éléments permanents du sursol (bâtiments, végétation). La visualisation par ombrage du MNS fournit une image proche d'une photographie aérienne (fig. 2a).

Du fait du couvert forestier, la plupart des techniques classiques de télédétection aérienne sont inopérantes pour révéler les vestiges qui y sont conservés. En revanche, grâce à une densité de points élevée (plusieurs milliers par hectare) et une pénétration du signal à l'intérieur du couvert forestier, les levés topographiques à l'aide d'un lidar constituent une méthode performante pour détecter les vestiges conservés sous forme de micro-reliefs. Il convient cependant pour cela de s'assurer d'une méthode de classification adéquate des points sol/sursol.

1. Dans notre cas, cette classification a été réalisée avec le logiciel Terrascan $^{\circ}$ de Terrasolid. 

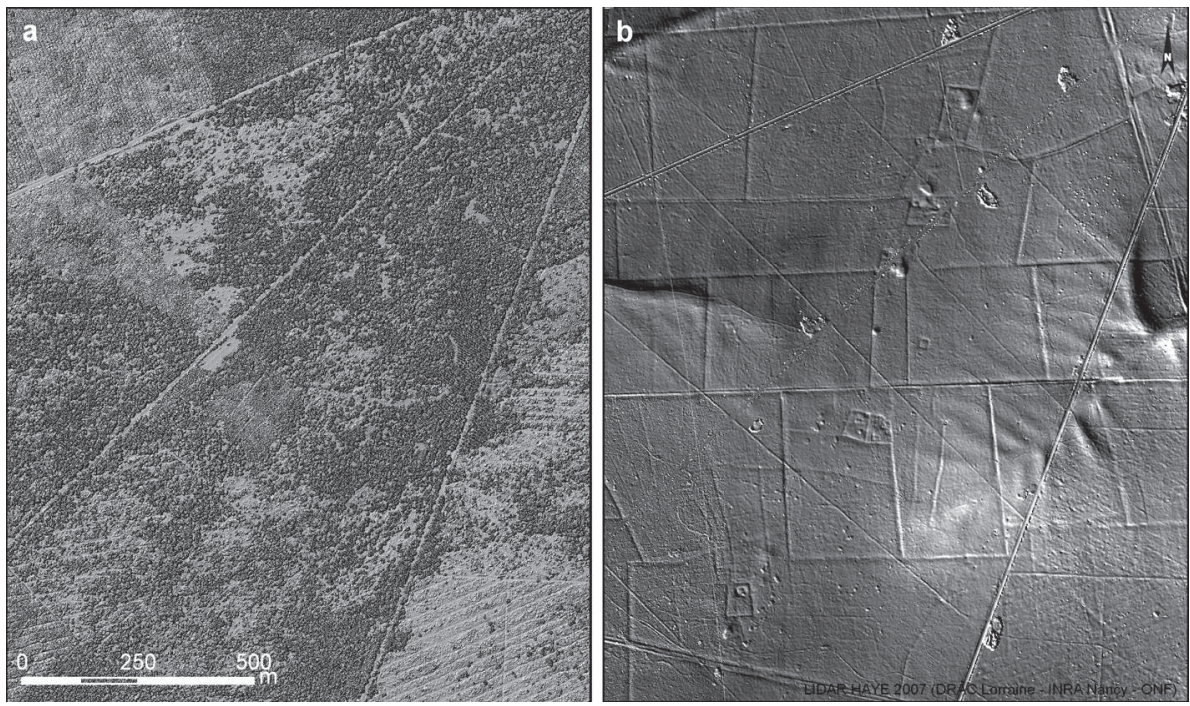

Figure 2 : Images comparatives d'un secteur de $1,8 \mathrm{~km}^{2}$ au sud du massif forestier de Haye obtenues a) à partir du MNS et b) à partir du MNT (ombrage : azimut $45^{\circ}$, altitude $6^{\circ}$ ). Outre les routes et chemins forestiers, bordés de carrières, on observe les parcellaires, les voies antiques, marquées par une double ligne de murées, et les habitats composés d'enclos entourant un ou plusieurs bâtiments.

Figure 2: Comparative images of a sector in the south of Haye Forest $\left(1.8 \mathrm{~km}^{2}\right)$ - Elevation models with (a) and without (b) vegetation. On the latter, in addition to modern roads and forest tracks, there are ancient track ways, marked by parallel stone banks, field boundaries and settlements composed of enclosures and buildings.

\section{Caractéristiques du vol de 2007 sur la forêt de Haye}

L'acquisition lidar au-dessus de la forêt de Haye, effectuée sur deux jours, a porté sur une surface totale de $116 \mathrm{~km}^{2}$, dont près de $5 \mathrm{~km}^{2}$ hors forêt. Elle a été réalisée par l'entreprise G2B/Geophenix, juste avant la reprise de la végétation, en mars 2007, avec un appareil laser Leica ALS50-II (tabl. 1). Quatre échos au maximum ont été mesurés par impulsion émise (1,5 en moyenne). La totalité de la zone a fait l'objet d'un levé avec une densité moyenne de 5,2 impulsions émises par mètre carré et un angle de balayage de $34^{\circ}$. Par ailleurs deux secteurs, d'une surface totale de $2,3 \mathrm{~km}^{2}$, ont fait l'objet d'un survol supplémentaire avec un angle de balayage de $18^{\circ}$ afin d'atteindre une plus grande densité de points $\left(10 \mathrm{pts} / \mathrm{m}^{2}\right)$, ce qui a permis de tester le rôle de la densité d'acquisition des points sur la qualité de la détection de structures archéologiques.

\section{Analyse des variations de densité de points sol}

Au final, c'est en moyenne 11,3 impulsions par mètre carré $^{2}$ qui ont été émises sur l'ensemble de la zone survolée. Cette "surdensité " par rapport à la densité théorique initiale s'explique par le recouvrement des bandes de vol et la réalisation d'un vol complémentaire nécessaire pour combler les manques sur certaines bandes (écarts trop importants par rapport au plan de vol initial). Ce sont environ 2 milliards d'échos qui ont été mesurés, dont 250 millions ont été classés en points "sol ", soit $19 \%$ des impulsions émises. Ce pourcentage représente en quelque sorte le "rendement " du vol lidar pour tous ceux qui s'intéressent au MNT. La

\begin{tabular}{|l|c|c|}
\hline \multicolumn{1}{|c|}{ Surface } & $116,3 \mathrm{~km}^{2}$ & $2,3 \mathrm{~km}^{2}$ \\
\hline Hauteur de vol & \multicolumn{2}{c|}{$700 \mathrm{~m}$} \\
\hline Vitesse de vol & $51,44 \mathrm{~m} / \mathrm{s}$ & $54,02 \mathrm{~m} / \mathrm{s}$ \\
\hline Fréquence des impulsions laser & $115,8 \mathrm{kHz}$ & $120,2 \mathrm{kHz}$ \\
\hline Fréquence de balayage & $49 \mathrm{~Hz}$ & $67,4 \mathrm{~Hz}$ \\
\hline Angle de balayage & $34^{\circ}\left(+/-17^{\circ}\right)$ & $18^{\circ}\left(+/-9^{\circ}\right)$ \\
\hline Densité moyenne de points laser & $5,26 \mathrm{pts} / \mathrm{m}^{2}$ & $10,04 \mathrm{pts} / \mathrm{m}^{2}$ \\
\hline Précision altimétrique des points laser mesurés (écart-type) & \multicolumn{2}{|c|}{$8 \mathrm{~cm}$} \\
\hline Précision planimétrique des points laser mesurés (écart-type) & \multicolumn{2}{|c|}{$25-30 \mathrm{~cm}$} \\
\hline
\end{tabular}

Tableau 1 : Paramètres d'acquisition lidar.

Table 1: Lidar acquisition parameters.
2. L'ensemble des calculs de densité de points a été réalisé avec le logiciel d'analyses statistiques SAS. 
densité moyenne des points sol après classification est de $2,11 \mathrm{pts} / \mathrm{m}^{2}$. Elle présente une très grande hétérogénéité, variant de 0 à plus de $10 \mathrm{pts} / \mathrm{m}^{2}$ (calculée sur des pixels de 25 x $25 \mathrm{~m}$ ). Cette variation est d'abord liée au mode d'acquisition lui-même et notamment aux zones de recouvrement entre les bandes de vol. Un constat important est que la densité de points sol, et donc la qualité des images utilisées pour l'interprétation archéologique ultérieure, n'augmente pas proportionnellement au nombre de passages de l'avion (tabl. 2). D'un point de vue purement numérique, le tableau 2 montre que la rentabilité d'un accroissement du temps de vol, en vue d'augmenter la densité de points sol, est globalement mauvaise et devient très mauvaise au-delà de deux passages.

Mais les types de peuplements forestiers (perméabilité du couvert) ont aussi une influence importante. Ainsi, dans les zones forestières, principalement constituées de feuillus, le taux de points classés sol par rapport au nombre d'impulsions émises (le " rendement " cité précédemment) est en moyenne de $40,3 \%$ pour un passage de l'avion, mais il n'est que de $36,7 \%$ dans les zones où la forêt a été totalement détruite par la tempête de 1999 et de 35,5\% dans les gaulis (peuplements forestiers denses compris entre 3 et $12 \mathrm{~m}$ ). Il atteint un minimum de $30 \%$ dans les peuplements de 5 à $6 \mathrm{~m}$ de hauteur. Dans les rares secteurs de conifères, on constate que les rayons pénètrent difficilement le couvert arboré. Le signal semble totalement intercepté par les houppiers des arbres et ne passe que dans les espaces qui les séparent.

Un autre résultat important est que, lors de ce vol effectué hors période feuillée, les rayons laser n'ont été que peu interceptés par les branches et les troncs des arbres. Nous avons calculé que $83 \%$ des impulsions émises par l'avion pénètrent jusqu’à la hauteur de $2 \mathrm{~m}$ au-dessus du sol (fig. 3 ), dans les peuplements de $4 \mathrm{~m}$ de hauteur et plus. C'est finalement dans la végétation arbustive basse, en dessous de $2 \mathrm{~m}$, qu'ont eu lieu ou ont été classés la majorité des impacts laser (64\%). Deux interprétations complémentaires peuvent être données à ce résultat :
- Le sous-bois, avec ses ronciers qui gardent en partie leurs feuilles en hiver, et ses fourrés de jeunes pousses denses peut en effet constituer un réel obstacle au passage du rayonnement lidar.

- Mais il se pourrait aussi que l'algorithme de classification employé conduise à rejeter en sursol une proportion importante des impacts réels au sol. Le fait que le nombre de points classés sol n'augmente que peu avec le nombre de passages de l'avion concorde avec cette dernière interprétation.

\section{Méthodes de traitements}

À l'issue du vol, les premiers traitements (classification sol/sursol, réalisation des MNT et MNE pour la végétation uniquement, sous forme de grilles au pas de $50 \mathrm{~cm}$ ) ont été réalisés par l'entreprise chargée de l'acquisition. Le niveau du filtrage retenu pour la classification a toutefois été discuté durant la phase d'élaboration de l'algorithme et validé par l'équipe archéologique. L'équipe s'est ainsi assurée que le filtrage n'était pas trop important et ne faisait pas disparaître d'éléments archéologiques, en se référant aux structures déjà connues et au système d'information géographique disponible initialement. Les autres traitements destinés à la visualisation des données ont été réalisés par l'équipe archéologique, avec le logiciel ArcGis 9.2 de ESRI et son extension 3DAnalyst. La visualisation la plus immédiate consiste à réaliser un ombrage à partir du MNT, en jouant sur l'altitude et les directions d'éclairage afin de faire apparaître l'ensemble des micro-reliefs (Devereux et al., 2008) (fig. 2b). En effet, certaines structures archéologiques n'apparaissent que sous une direction d'éclairage particulière. Pour les zones les plus planes on a utilisé un ombrage très rasant, à une altitude de $3^{\circ}$, et deux azimuts $\left(315^{\circ}\right.$ et $\left.45^{\circ}\right)$. Dans les zones pentues, la visualisation des micro-reliefs par cette méthode est plus délicate, car ils sont masqués par la pente naturelle. Nous avons donc dû parfois utiliser des éclairages un peu moins rasants, à une altitude de $6^{\circ}$ ou $10^{\circ}$ et, en complément des deux azimuts principaux, nous avons utilisé des azimuts à $225^{\circ}$ et $135^{\circ}$.

\begin{tabular}{|c|c|c|c|c|c|}
\hline Nombre de passages de l'avion & 1 & 2 & 3 & 4 & total \\
\hline Surface analysée $\left(\mathrm{km}^{2}\right)$ & 18,6 & 48,8 & 36,1 & 3,8 & 107,5 \\
\hline Nombre moyen d'impulsions émises par $\mathrm{m}^{2}$ & 4,1 & 10,0 & 15,8 & 20,8 & 11,3 \\
\hline Nombre moyen de points classés sol par $\mathrm{m}^{2}$ & 1,6 & 2,1 & 2,3 & 2,4 & 2,1 \\
\hline Taux de points classés sol par rapport au nombre d'impulsions émises (\%) & 40 & 21 & 15 & 12 & 19 \\
\hline
\end{tabular}

Tableau 2 : Relation entre nombre de survols, impulsions envoyées et points classés en sol.

Table 2: Comparison between number of overflights, laser pulses and points classified into ground. 


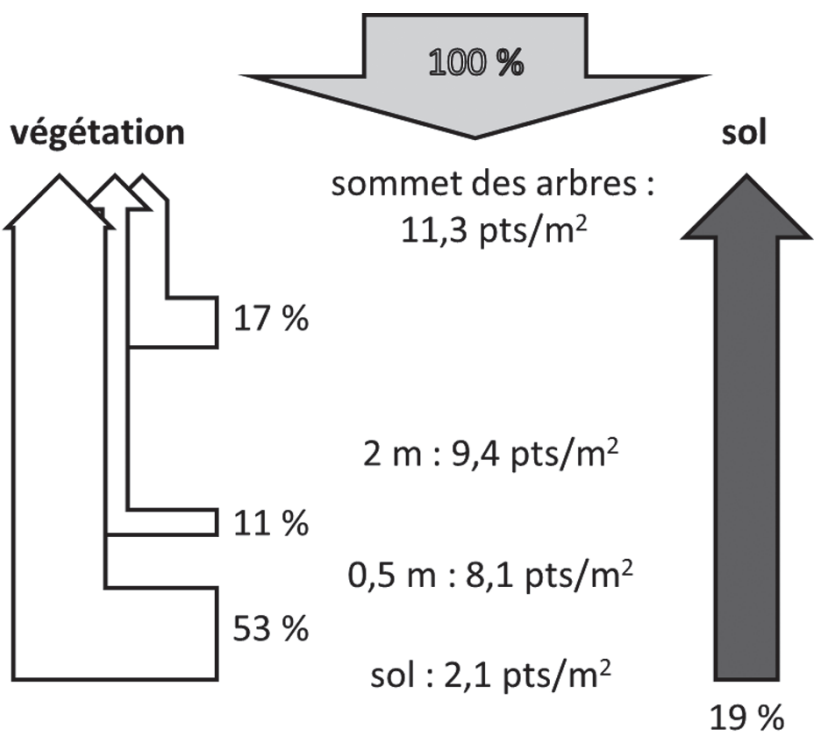

Figure 3 : Pénétration du signal lidar en fonction de la hauteur dans le peuplement.

Figure 3: Lidar penetration in woodland vegetation as a function of stand height.

Même si le résultat est souvent spectaculaire, on ne peut pas se contenter de ces images pour l'analyse et l'interprétation des vestiges qui nous intéressent. Une étape fondamentale pour permettre cette analyse consiste à définir les objets archéologiques que l'on souhaite cartographier et le protocole de vectorisation que l'on va utiliser. Cette approche est encore souvent absente dans les études de données lidar en archéologie, dans lesquelles on se limite à la seule production d'images. Dans notre cas, plusieurs objets archéologiques ont ainsi été retenus (structure agraire et parcellaire, bâtiment, chaussée, tas, rempart, limite forestière), mais aussi des éléments non archéologiques, nécessaires à la compréhension des vestiges (zones détruites par des aménagements récents, zones perturbées par des effondrements miniers ou des aménagements militaires). Chaque objet archéologique a été défini morphologiquement et des normes de cartographie ont été précisément déterminées (tabl. 3). La datation des différents éléments a été renseignée dans un second temps, en croisant les données de terrain (mobilier recueilli), les données historiques (analyse des cartes anciennes) et les données lidar (analyse des connexions, morphologie des structures, etc.). Ces objets archéologiques de base ont ensuite servi à la construction d'objets plus complexes et d'un niveau d'interprétation plus élevé. Ainsi, les voies antiques qui se présentent sous deux formes - des voies principales composées d'une chaussée centrale éventuellement bordée de levées latérales et de fossés; des voies secondaires maté- rialisées par une double ligne de levées - sont construites à partir des objets " parcellaire " et " chaussée ». Les habitats, qui se présentent comme des bâtiments isolés ou comme des enclos entourant des bâtiments, sont quant à eux construits à partir des objets « bâtiment » et " parcellaire » et grâce aux prospections de terrain. L'essentiel du travail a porté sur les structures agraires et parcellaires qui sont notamment utilisées dans les travaux d'analyse spatiale menés dans le projet ArchaeDyn. Celui-ci a pour but de mettre en évidence et de caractériser les discontinuités spatiales dans l'utilisation ancienne du sol, au travers de la variation de l'intensité et de la structuration de ces parcellaires fossiles (Georges-Leroy et al., 2008).

Afin de limiter les imprécisions inhérentes aux images ombrées (ombrage porté, taille de cellule de l'image de $50 \mathrm{~cm}$ ), leur cartographie a été réalisée en superposant des courbes de niveau espacées de 5 à $15 \mathrm{~cm}$ à ces ombrages(fig. 4). Les structures ont pu être ainsi dessinées précisément sur le sommet des levées ou la rupture de pente du talus. Associées à des profils réalisés à travers les structures, les courbes de niveau permettent d'identifier la morphologie de ces structures (talus ou levée), qui n'apparaît pas sur les images ombrées. Ce travail requiert un apprentissage dans la lecture et l'analyse des images, avec l'élaboration de référentiels, afin de différencier les vestiges archéologiques des structures liées à l'exploitation forestière par exemple (andains, cloisonnements forestiers, etc.), mais aussi pour distinguer les différents types de structures entre elles. L'importante base de relevés dont nous disposions au départ, ainsi que les vérifications de terrain, ont permis la validation de ces référentiels. Il faut enfin insister sur le fait que, si la phase d'acquisition des données et les premiers traitements sont assez rapides à réaliser, hormis du temps de calcul pour les ordinateurs, la phase d'analyse et de cartographie, avec retour sur le terrain, nécessite un investissement humain en temps beaucoup plus important.

\section{RÉSUltats}

\section{Un apport considérable à la connaissance des structures agraires et parcellaires}

Le scanneur laser a permis de cartographier $647 \mathrm{~km}$ linéaires de vestiges agraires et parcellaires de toutes époques sur $116 \mathrm{~km}^{2}$ (fig. 5a). Ces chiffres sont à comparer avec les 275 km qui avaient été relevés entre 1979 et 2006 avec les autres moyens techniques sur une surface d'environ $75 \mathrm{~km}^{2}$ (fig. 5b). Ces structures linéaires, même celles qui sont très peu élevées et quasi imperceptibles à l'œil sur le terrain, 


\begin{tabular}{|c|c|c|c|}
\hline Objet archéologique & Définition morphologique & Normes de cartographie & $\begin{array}{l}\text { Nombre d'entités } \\
\text { cartographiées }\end{array}$ \\
\hline $\begin{array}{c}\text { Parcellaire } \\
\text { (structure agraire et/ } \\
\text { ou parcellaire) }\end{array}$ & $\begin{array}{l}\text { Linéaires, en général de grande extension, } \\
\text { pour lesquels } 3 \text { types ont été distingués : } \\
\text { levée, talus simple, talus avec levée. } \\
\text { Les petits enclos avec bâtiments sont inclus } \\
\text { dans cette catégorie. }\end{array}$ & $\begin{array}{l}\text { Polylignes } \\
-1 \text { entité par type et à chaque changement } \\
\text { d'orientation supérieur à } 45^{\circ} \\
\text { - elles sont cartographiées sur le sommet de } \\
\text { la levée ou légèrement en amont de la rupture } \\
\text { de pente }(c f . \text { fig. } 4)\end{array}$ & $\begin{array}{l}5924 \\
\text { (soit une longueur } \\
\text { totale de } 647296 \mathrm{~m} \text { ) }\end{array}$ \\
\hline Chaussée & $\begin{array}{l}\text { Chaussée d'une voie principale qui se } \\
\text { présente sous } 3 \text { formes : en butte, en terrasse, } \\
\text { en creux. } \\
\text { La chaussée peut être bordée de dispositifs } \\
\text { latéraux (levée ou talus) qui sont } \\
\text { cartographiés comme "parcellaire " et de } \\
\text { fossés qui ne sont pas cartographiés. } \\
\text { Ces chaussées datent toutes de l'Antiquité. }\end{array}$ & $\begin{array}{l}\text { Polygones } \\
\text { Les limites peuvent correspondre, selon les } \\
\text { cas : } \\
\text { - au fond du fossé s'il existe, } \\
\text { - à l'emprise du creux quand la voie est en } \\
\text { creux, } \\
\text { - aux ruptures de pente quand elle est en } \\
\text { terrasse, } \\
\text { - à la butte qu' elle forme. } \\
\end{array}$ & $\begin{array}{l}65 \\
\text { (sur une longueur } \\
\text { totale d'environ } \\
16960 \mathrm{~m} \text { ) }\end{array}$ \\
\hline Bâtiment & $\begin{array}{l}\text { Se présente soit comme : } \\
\text { - une forme le plus souvent fermée, délimitée } \\
\text { par des levées droites ou en arc de cercle, avec } \\
\text { des angles droits dominants, } \\
\text { - un monticule de forme rectangulaire. } \\
\text { Surfaces variant de } 35 \text { à } 500 \mathrm{~m}^{2} \text {. }\end{array}$ & $\begin{array}{l}\text { Polygones } \\
\text { Les limites sont cartographiées sur le sommet } \\
\text { du monticule ou des levées et non à leur base. }\end{array}$ & $\begin{array}{l}93 \\
\text { (soit une surface totale } \\
\text { de } 12376 \mathrm{~m}^{2} \text { ) }\end{array}$ \\
\hline $\begin{array}{l}\text { Tas } \\
\text { (épierrement, } \\
\text { tumulus) }\end{array}$ & Tas ou monticules de forme circulaire. & Points & 144 \\
\hline Rempart & $\begin{array}{l}\text { Fortification composée soit : } \\
\text { - d'une levée importante associée à un fossé, } \\
\text { - d'une faible levée bordant une rupture de } \\
\text { pente naturelle. } \\
\text { Ces fortifications sont attribuées à l'époque } \\
\text { protohistorique }\end{array}$ & $\begin{array}{l}\text { Polylignes } \\
\text { Les limites sont cartographiées sur le sommet } \\
\text { de la levée. }\end{array}$ & $\begin{array}{l}4 \text { sites (soit une } \\
\text { longueur totale de } \\
4252 \mathrm{~m} \text { ) }\end{array}$ \\
\hline $\begin{array}{l}\text { Fossé/butte de limite } \\
\text { forestière } \\
\text { (woodbanks sensu } \\
\text { Szabó, 2010) }\end{array}$ & $\begin{array}{l}\text { Dispositif composé d'une levée de terre } \\
\text { et d'un fossé associé, le fossé étant le plus } \\
\text { souvent en position externe par rapport au } \\
\text { massif forestier. }\end{array}$ & $\begin{array}{l}\text { Polylignes } \\
\text { Elles sont cartographiées sur le sommet de la } \\
\text { levée. }\end{array}$ & $\begin{array}{l}176 \\
\text { (soit une longueur } \\
\text { totale de } 35308 \mathrm{~m} \text { ) }\end{array}$ \\
\hline
\end{tabular}

Tableau 3 : Objets archéologiques cartographiés d'après le levé lidar. Table 3: System of reference of archaeological objects identified in lidar survey.

apparaissent remarquablement bien sur les images lidar, y compris dans les zones totalement détruites par la tempête de 1999 et même dans une zone où les arbres n'ont pas été dégagés depuis cette date. Ces images permettent en effet un recul que l'on n'a que rarement en forêt à cause de la végétation. La cartographie a donc pu être réalisée dans les zones non étudiées auparavant, mais également être complétée dans les secteurs déjà étudiés. Ainsi dans les zones considérées précédemment comme bien prospectées au sol, on a découvert $50 \%$ de vestiges supplémentaires. Dans les zones déjà étudiées, mais qui posaient des problèmes d'accessibilité dus au type de peuplement forestier (jeunes peuplements, zones de tempête), la longueur des vestiges cartographiés a été multipliée par 2,3. Par ailleurs, si l'accessibilité joue un rôle important dans le repérage au sol des vestiges, ce repérage est aussi tributaire de leur état de conservation. Ainsi, dans un secteur pourtant bien prospecté, mais où les vestiges sont très peu perceptibles au sol, la longueur cartographiée par lidar a été multipliée pratiquement par quatre. En revanche, il semble que la grande variabilité de la densité des points sol n'influence pas le nombre de structures nouvelles découvertes; les variations à courte distance de cette densité étant probablement sans effet pour des structures linéaires d'une certaine longueur.

L'apport quantitatif du lidar par rapport aux méthodes classiques de prospection au sol est donc tout à fait considérable, notamment quand on approche les limites de perception de l'œil humain : pour les vestiges les plus discrets et dans les milieux forestiers les moins accessibles ou les moins visibles. De plus, ce levé lidar a permis de corriger les erreurs 


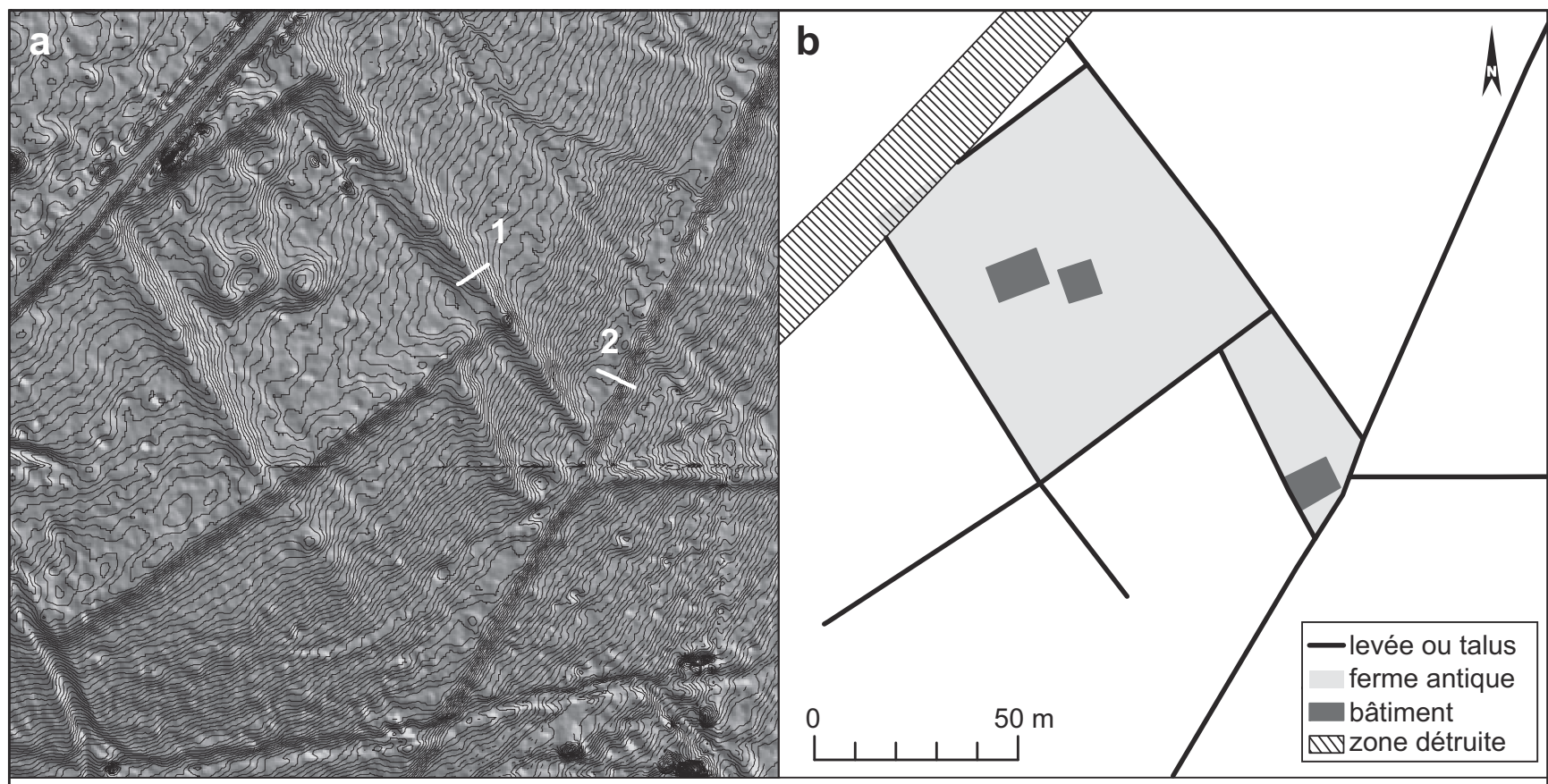

C
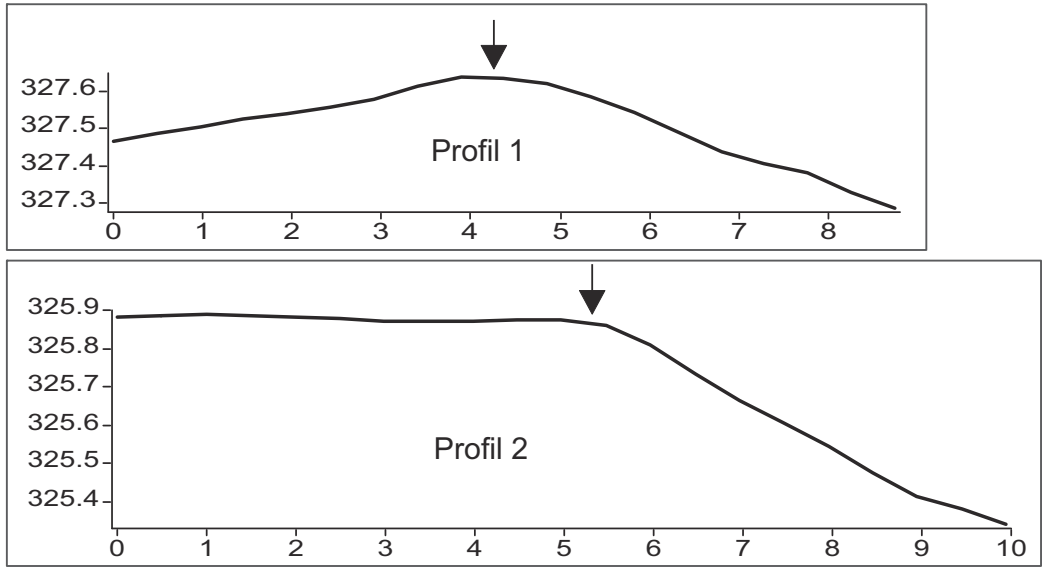

Figure 4 : Ferme antique : a) image lidar ombrée avec courbes de niveaux (5 cm d'équidistance); b) cartographie des vestiges antiques; c) profils à travers une levée de pierres (1) et un talus (2).

Figure 4: Antique farm: a) lidar hillshade image with contour lines (equidistance: $5 \mathrm{~cm}$ ); b) map of antique remains; c) elevation profiles across: (1) a stone row, (2) a bank.

de positionnement souvent importantes des vestiges relevés initialement à la boussole et au décamètre ou au topofil. Des écarts jusqu'à $90 \mathrm{~m}$ ont ainsi pu être mesurés. La comparaison avec les données cartographiées au GPS différentiel ${ }^{3}$ a en revanche montré une assez bonne précision du GPS sous couvert forestier, puisque la très grande majorité de ces données ne sont pas éloignées de plus de $5 \mathrm{~m}$ de celles

3. Les GPS utilisés sont des Trimble ProXL, Pathfinder Power et GeoXT. acquises par lidar. La correction de ces erreurs de positionnement, associée à la cartographie des nouveaux tronçons, révèle ainsi un parcellaire beaucoup plus dense et cohérent qu'il n'y paraissait auparavant.

Enfin, outre l'intérêt démontré du scanneur laser pour augmenter le nombre de linéaments cartographiés et améliorer leur localisation, cette méthode a également permis une approche morphologique de ces vestiges. Ainsi, grâce à la lecture des images lidar, il est possible de repérer certaines 


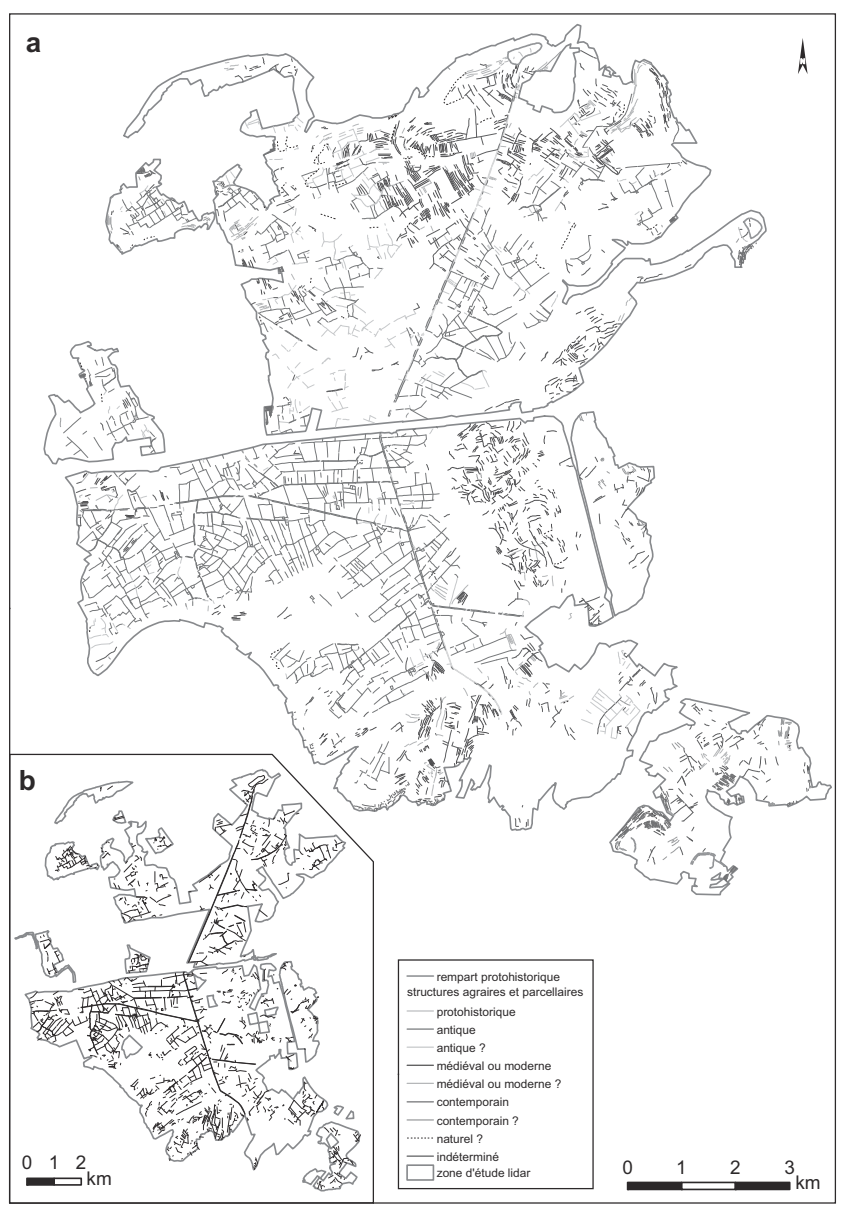

Figure 5: (Voir planche couleur) Cartographie des structures agraires et parcellaires de la forêt de Haye : a) relevés réalisés à partir du levé lidar de 2007; b) relevés réalisés au sol de 1979 à 2006. Figure 5: (See colour plate) Map of agricultural structures and field systems of Haye Forest: a) from lidar survey of 2007; b) from ground surveys conducted between 1979 and 2006.

traces agraires plutôt typiques de l'utilisation de la charrue : billons ou rideaux de culture curvilignes, associés à des crêtes de labour (fig. 6c). Elles sont donc postérieures à l'époque gallo-romaine, alors que les autres structures agraires et parcellaires sont attribuées à l'Antiquité, grâce à leur connexion avec des habitats datés par du mobilier antique. Or, sur le terrain, ces structures médiévales ou modernes ne se distinguent pas morphologiquement des structures antiques, à l'exception du fait que certaines sont beaucoup moins perceptibles. Elles témoignent de défrichements de la forêt postérieurs à l'époque gallo-romaine et antérieurs pour certaines au XVII ${ }^{\mathrm{e}}$ siècle comme le montre l'analyse des cartes anciennes disponibles sur le secteur (Georges-Leroy et al., 2010). Nous avions minimisé ces faits avant la télédétection par scanneur laser, faute de bien les appréhender.

\section{De nouvelles données sur les habitats antiques}

Le résultat est également très positif pour les habitats, qui se présentent soit sous la forme de fermes à enclos entourant un ou plusieurs bâtiments, soit sous la forme de bâtiments isolés. Leur surface varie de quelques dizaines de mètres carrés pour les bâtiments isolés jusqu'à plusieurs milliers de mètres carrés pour les habitats à enclos (Georges-Leroy et al., sous presse; Fig. 4). Sur les 85 habitats identifiés actuellement dans le massif forestier, 26 soit $30 \%$ ont été découverts grâce au scanneur laser et confirmés par prospection au sol. 24 de ces nouveaux habitats sont localisés dans des zones au moins partiellement prospectées auparavant, mais en grande majorité dans des secteurs où la visibilité était réduite du fait de la végétation. Il s'agit principalement d'habitats à enclos, mais aussi de quatre bâtiments isolés, d'une surface supérieure à $100 \mathrm{~m}^{2}$. De plus, 21 habitats déjà connus ont vu leur connaissance améliorée ou même totalement renouvelée. Les apports ont surtout concerné les enclos, mais quelques nouveaux bâtiments ont aussi été identifiés.

Concernant les bâtiments, sur un effectif total de 93 bâtiments, dont 35 sont d'interprétation incertaine, le lidar a permis la découverte de 31 d'entre eux dont 15 incertains. Les plus petits de ces bâtiments ont des surfaces inférieures à $50 \mathrm{~m}^{2}$. En revanche, quatre bâtiments isolés ne sont pratiquement pas visibles sur les images lidar, mais ils sont également très peu lisibles sur le terrain car localisés dans des zones détruites par la tempête.

Par ailleurs, il n'existe pas de corrélation entre la découverte ou l'amélioration des connaissances sur les habitats et la densité des points sol, qui varie à l'emplacement des habitats de 0,95 à $3,87 \mathrm{pts} / \mathrm{m}^{2}$. Et si l'on examine le cas du seul habitat couvert par les deux densités de vol, soit des densités respectives de points sol de 2,05 et $3,14 \mathrm{pts} / \mathrm{m}^{2}$, on constate que la résolution de l'image est certes plus fine et donc plus lisible, mais aucun nouvel élément d'enclos ou bâtiment n'est observable sur les images à plus forte densité. Mais il ne s'agit là que d'un exemple, localisé de plus dans une zone très touchée par la tempête de 1999 et non exploitée depuis, ce qui nuit à sa perception générale.

Enfin, la taille minimale des vestiges repérables avec les paramètres de vol utilisés varie en fonction de leur type. Ainsi, les plus petits vestiges en élévation perceptibles sont des tas de pierres circulaires (probables tas d'épierrement), de 6 à $8 \mathrm{~m}$ de diamètre et de 20 à $30 \mathrm{~cm}$ de hauteur. En revanche, les structures en creux sont perceptibles à des tailles inférieures : on distingue en effet très bien sur les images des creusements circulaires, de 2 à $3 \mathrm{~m}$ de diamètre et d'une cinquantaine de centimètres de profondeur. Il est possible que cette différence de seuil de détection entre les 


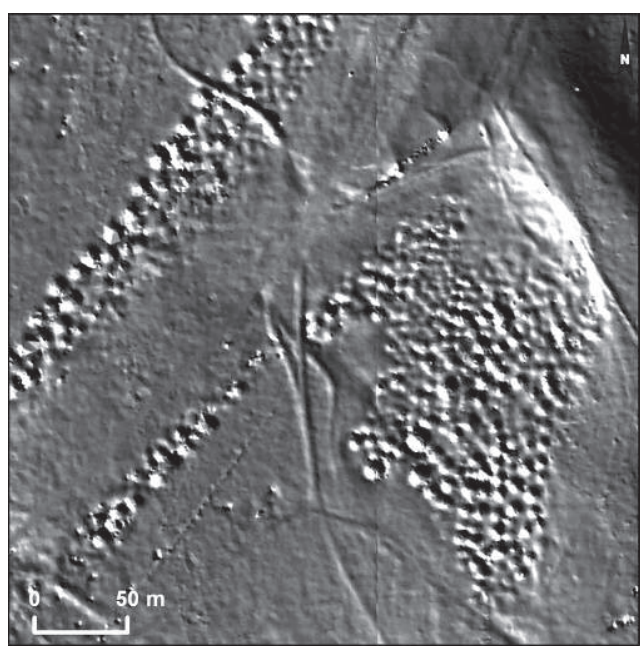

a. Minières de fer d'époque médiévale.

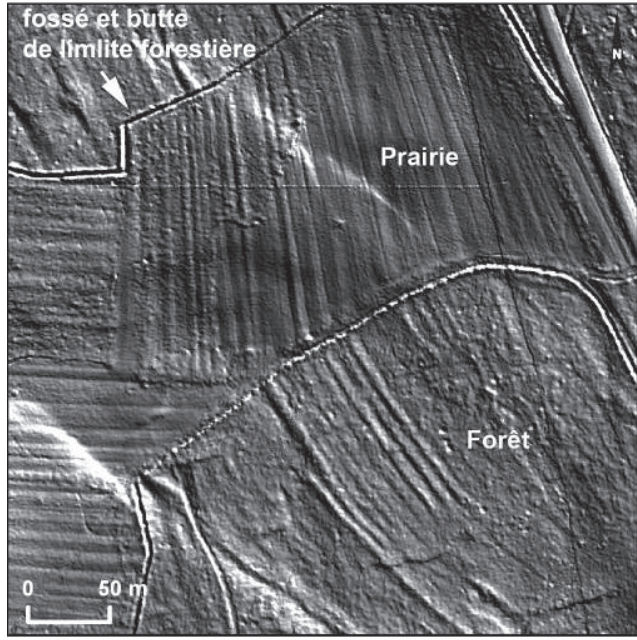

c. Structures agraires : médiévales ou modernes (billons et crêtes de labour) en forêt, contemporaines

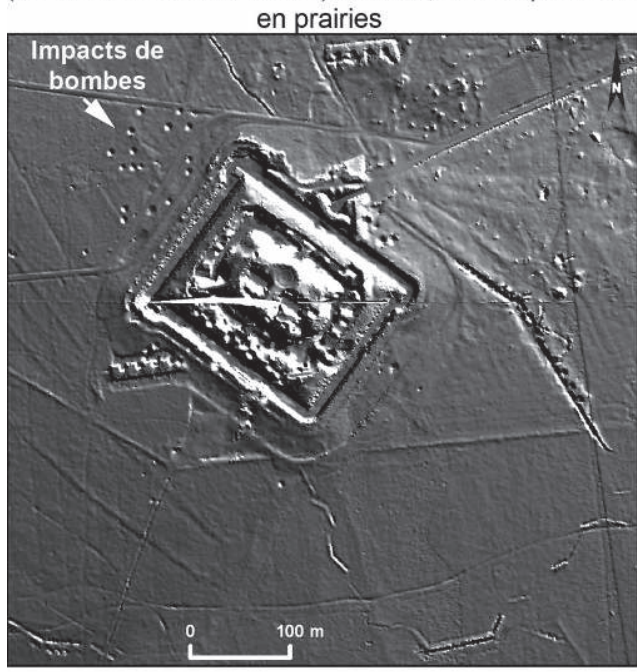

e. Fort de Frouard et aménagements militaires divers (batteries, tranchées) du XIXe siècle

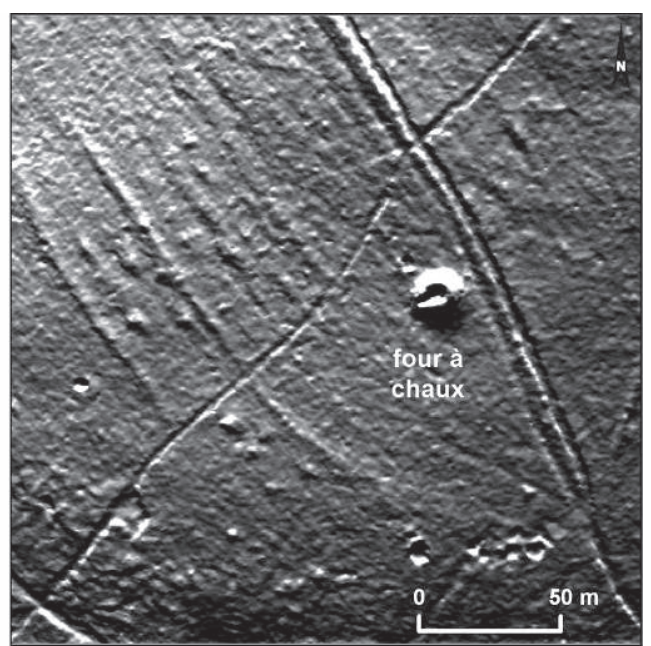

b. Four à chaux et structures agraires et parcellaires (tas, talus, murées)

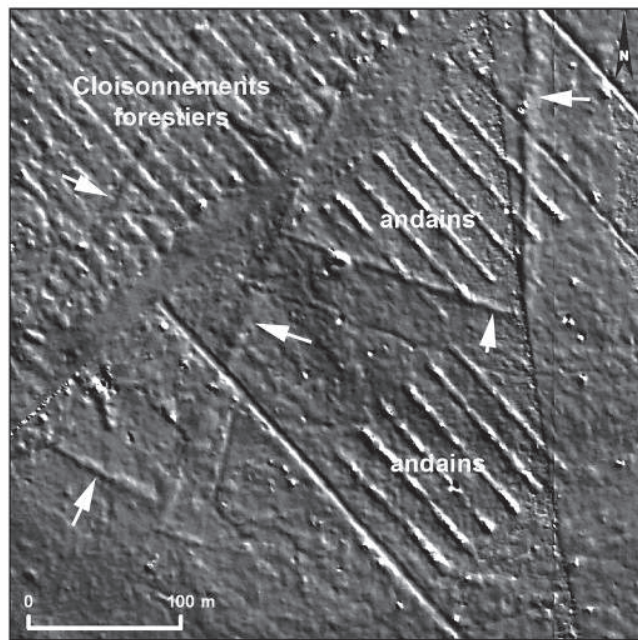

d. Parcellaires antiques (flèches) et structures liées à la forêt (andains, cloisonnements)

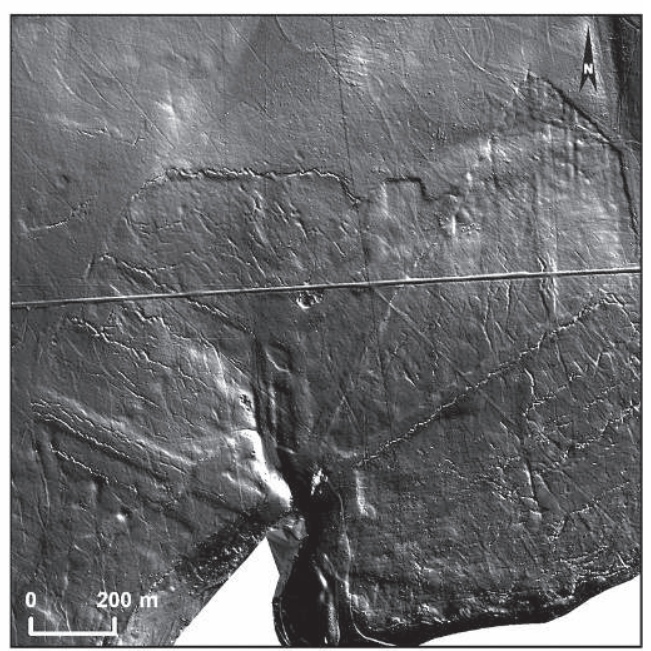

f. Zone d'effondrements miniers du XXe siècle.
Figure 6 : Quelques exemples de vestiges archéologiques et historiques et de phénomènes divers (images lidar ombrées).

Figure 6: Some archaeological and historical remains identified on lidar hillshade images: a) medieval iron mines; b) lime kiln; c) modern and medieval fields (ridge and furrow associated with headland ridge); d) Gallo-Roman field systems and forest management structures (windrows); e) $19^{\text {th }}$ century military fort; f) $20^{\text {th }}$ century mine subsidence. 
vestiges en creux et en bosses soit attribuable à l'algorithme de classification utilisé dans ce projet. Des structures plus petites sont encore visibles sur les images, mais elles ne sont plus assez détaillées pour être identifiées correctement.

Comme pour les structures agraires et parcellaires, l'apport quantitatif du lidar pour les habitats par rapport aux méthodes de prospection traditionnelles semble donc important dans les milieux forestiers les plus difficiles. De plus, de nouveaux habitats ont été découverts dans des secteurs qui avaient été considérés comme bien prospectés et ne présentaient pas de difficultés d'accès particulières. Cela montre que lorsque la zone de prospection atteint plusieurs milliers d'hectares et qu'il n'est pas possible de procéder à des prospections au sol véritablement systématiques (de type prospection en lignes), des vestiges de quelques dizaines à quelques milliers de mètres carrés peuvent facilement être "manqués".

Le lidar renouvelle donc nos connaissances sur ces habitats antiques. D'une part, grâce à cet inventaire beaucoup plus exhaustif, il permet une approche sur leur répartition spatiale, au moins dans les secteurs non perturbés par des aménagements postérieurs (aménagements militaires, effondrements miniers, secteurs remis en culture au Moyen Âge ou à l'Époque Moderne). D'autre part, il nuance la perception globale que l'on avait auparavant de leur morphologie, notamment en augmentant la part des habitats à enclos.

\section{Élaboration d'un référentiel d'interprétation}

En plus des habitats et parcellaires antiques et des structures agraires médiévales ou modernes, le massif forestier de Haye recèle de nombreux autres vestiges archéologiques, dont un grand nombre sont visibles sur les images lidar (Georges-Leroy et al., 2010). Ainsi on y recense plusieurs sites de hauteur fortifiés protohistoriques, "Côte du Pimont " à Frouard, "La Fourasse " à Champigneulles, "Camp d'Affrique " à Messein, "Bois de Châtel " à Chavigny. Ce dernier est un site inédit découvert sur les images lidar, alors même que ce secteur a fait l'objet de nombreuses prospections au XIX ${ }^{e}$ et au début du $\mathrm{Xx}^{\mathrm{e}}$ siècle. Les structures conservées en élévation à l'intérieur du "Camp d'Affrique " ont, quant à elles, fait l'objet d'une nouvelle analyse grâce au levé lidar (Deffressigne et Tikonoff, soumis). Dans le massif forestier, ont également été repérés des vestiges d'extraction du minerai de fer, des fours à chaux et des carrières d'extraction du calcaire, des tranchées de chasse d'Époque Moderne, de nombreux chemins creux de toutes époques, mais aussi de nombreux vestiges militaires de la fin du XIX et du début du Xx ${ }^{\mathrm{e}}$ siècle (forts, batteries, etc.) (fig. $6 a, b, e)$. Cette richesse archéologique et notre bonne connaissance de cette forêt nous ont permis d'élaborer à partir des images lidar un catalogue de vestiges archéologiques, mais aussi de structures liées à la forêt (andains, cloisonnements, chablis - fig. 6d) ou de phénomènes divers (effondrements miniers par exemple - fig. 6f). Ce catalogue, outil de référence nécessaire à l'identification de ces diverses structures, pourra être réutilisé sur de nouveaux secteurs où nous ne connaissons pas, ou beaucoup moins, les vestiges présents.

\section{ConClusion}

L’apport de la télédétection par scanneur laser réalisée sur le massif forestier de Haye est donc considérable, tant par la découverte de nouveaux vestiges que par l'amélioration de leur connaissance. Pendant longtemps, la difficulté à percevoir et à cartographier les parcellaires en forêt a été un frein à leur étude et, en l'absence de cette cartographie, on en a minimisé l'ampleur. Le lidar permet un véritable changement d'échelle dans l'étude des sites archéologiques en révélant, dans le cas du massif forestier de Haye, un ensemble de parcellaires fossilisé sur plus de 11000 ha, ce qui représente une superficie considérable. À titre de comparaison, les emblématiques parcellaires de l'Âge du Bronze fossilisés sous les landes du Dartmoor (Grande-Bretagne), couvrent une surface équivalente : les reaves, qui correspondent à nos murées, s'étendent sur plus de 10000 ha, sur un territoire d'environ $35 \mathrm{~km}$ par $23 \mathrm{~km}$ (Fleming, 2008).

Par ailleurs, cette cartographie, associée à la datation des vestiges agraires et parcellaires, permet d'esquisser dans ses grandes lignes l'histoire de l'occupation du sol de l'actuel massif forestier de Haye au moins depuis 2000 ans (Georges-Leroy et al., 2010). Ainsi ce plateau calcaire a été presque entièrement défriché et mis en culture au moins à partir de l'époque gallo-romaine. Puis la forêt a reconquis probablement tout ou partie de l'espace jusqu'à nos jours, en connaissant toutefois une ou plusieurs autres phases de défrichement importantes dans plusieurs zones. Ces données illustrent donc les importantes fluctuations dans le mode d'exploitation qu'a connu ce terroir et montrent que la couverture forestière n'a pas seulement évolué selon des pulsations de déboisement/reboisement autour de noyaux pérennes, mais s'est bien déplacée dans le territoire, selon des modalités encore à préciser.

Grâce à la méthode de cartographie mise au point et au référentiel acquis lors de cette étude, il est maintenant envisageable d'engager une prospection lidar à une échelle encore plus importante, en abordant la totalité des massifs forestiers du plateau bajocien, au moins entre Nancy et Neufchâteau, 
où de nombreux parcellaires antiques restent à inventorier et cartographier. Une telle échelle nous permettra peut-être de comprendre pourquoi ces espaces agraires massivement cultivés durant l'Antiquité sont depuis retournés à la forêt.

\section{Remerciements}

Ce programme a bénéficié du financement de la Région Lorraine, de la Direction Régionale des Affaires Culturelles de Lorraine, de l'Institut National de Recherche Agronomique et de l'Office National des Forêts.

Un grand merci à Patrick Behr pour son concours dans le montage technique de cette télédétection par scanneur laser aéroporté et à Alexia Fontaine pour sa participation à la modélisation des objets archéologiques et à la cartographie des structures agraires et parcellaires.

\section{Bibliographie}

Bewley, R. H., Crutchley, S. P. et Shell, C. A., 2005. New light on an ancient landscape: lidar survey in the Stonehenge World Heritage Site, Antiquity, 79, p. 636-647.

Bilodeau, C. et Deroin, J.-P., 2008. Le principe du LiDAR, son utilisation et ses limitations en archéologie, Archéopages, 23, p. 65-68.

Deffressigne, S. et Tikonoff, N., avec la coll. de GeorgesLeroy, M., soumis. La proto-urbanisation dans le bassin de Nancy (Lorraine) de la fin du vi ${ }^{\mathrm{e}}$ au I ${ }^{\mathrm{er}}$ siècle avant J.-C, Actes du $34^{e}$ colloque international de l'AFEAF. 13 au 16 mai 2010. Aschaffenburg.

Devereux, B. J., Amable, G. S. et Crow, P., 2008. Visualisation of LiDAR terrain models for archaeological feature detection, Antiquity, p. 470-479.

Devereux, B. J., Amable, G. S., Crow, P. et Cliff, A. D., 2005. The potential of airborne lidar for detection of archaeological features under woodland canopies, Antiquity, 79, p. 648-660.

Doneus, M., Briese, C., Fera, M. et Janner, M., 2008. Archaeological prospection of forested areas using full-waveform airborne laser scanning, Journal of Archaeological Science, p. 882-893.

Dupouey, J.-L., Dambrine, E., Laffite, J.-D. et Moares, C., 2002. Irreversible impact of past land use on forest soils and biodiversity, Ecology, 83, p. 2978-2984.

Dupouey, J.-L., Sciama, D., Laffite, J.-D., Georges-Leroy, M. et Dambrine, E. 2007. Impact des usages agricoles antiques sur la végétation en forêt de Saint-Amond : interaction avec le traitement sylvicole actuel, dans J.-L. Dupouey, E. Dambrine, C. Dardignac, M. Georges-Leroy (éd.). La mémoire des forêts.
Actes du colloque "Forêt, Archéologie et Environnement " 14-16 décembre 2004, ONF - INRA - DRAC Lorraine, p. 181-189.

Fleming, A., 2008. The Dartmoor Reaves. Investigating prehistoric land divisions. $2^{e}$ edition, Windgather Press, Oxford, $224 \mathrm{p}$.

Georges-Leroy, M., Dambrine, E., Dupouey, J.-L., Laffite, J.-D., 2003. Habitats gallo-romains et structures agraires fossiles du plateau de la Côte bajocienne (Meurthe-et-Moselle et Vosges). Etat de la question, dans F. Favory, A. Vignot (dir.). Actualité de la recherche en histoire et archéologie agraires. Actes du colloque international AGER V - 19-20 septembre 2000, Besançon, Presses Universitaires Franc-Comtoises, coll. "Annales Littéraires; 764 », p. 173-180.

Georges-Leroy, M., Heckenbenner, D., Laffite, J.-D., Meyer, N., Dambrine, E. et Dupouey, J.-L., 2007. Les parcellaires anciens fossilisés dans les forêts lorraines, dans J.-L. Dupouey, E. Dambrine, C. Dardignac, M. Georges-Leroy (éd.), La mémoire des forêts. Actes du colloque "Forêt, Archéologie et Environnement" 14-16 décembre 2004. ONF - INRA DRAC Lorraine, p. 121-131.

Georges-Leroy, M., Tolle, F. et Nouvel, P., 2008. Analysis of the intensity of agrarian exploitation by spatial analysis of ancient field systems preserved by forest cover, dans A. Posluschny, K. Lambers, I. Herzog (ed). Layers of perception. Proceedings of the $35^{\text {th }}$ International Conference on Computer Applications and Quantitative Methods in Archaeology (CAA). Berlin, Germany, April 2-6, 2007. Habelt, Bonn, 281 (abstract) \& full paper on CD (Kolloquien zur Vor- und Frühgeschichte 10).

Georges-Leroy, M., Bock, J., Dambrine, E. et Dupouey, J.-L., 2010. Le massif forestier, objet pertinent pour la recherche archéologique. L'exemple du massif forestier de Haye (Meurthe-et-Moselle), Revue Géographique de l'Est [En ligne], 49/2-3, 2009, Le massif forestier, objet géographique, mis en ligne le 7 octobre 2010, consulté le 14 octobre 2010 [http:// rge.revues.org/1931].

Georges-Leroy, M., Bock, J., Dambrine, E., Dupouey, J.-L., GEBHARDT, A. et LAFFITE, J.-D., sous presse. Les vestiges galloromains conservés dans le massif forestier de Haye (Meurtheet-Moselle) : leur apport à l'étude de l'espace agraire, dans Actes de la table-ronde "Des hommes aux champs " 8-9 octobre 2008 Caen, Presses universitaires de Rennes.

Gallagher, J. M. et Josephs, R. L., 2008. Using LiDAR to detect cultural resources in a forested environment: an example from Isle Royale National Park, Michigan, USA, Archaeological Prospection, 15, p. 187-206.

Humme, A., Lindenbergh, R. et Sueur, C., 2006. Revealing Celtic fields from Lidar data using kriging based filtering. In H.-G. Maas et D. Schneider (ed.), Proceedings of the ISPRS Commission $V$ Symposium 'Image Engineering and Vision Metrology' Dresden 2006. 
Joinville, O. De, Saur, S. et Bretar, F., 2003. Le levé laser aéroporté : techniques, applications et recherche, Bulletin d'information scientifique et technique de l'IGN, 74 (2003/3), p. 37-52.

Kooistra, M. J. et MaAS, G. J., 2008. The widespread occurrence of Celtic field systems in the central part of the Netherlands. Journal of Archaeological Science, 35, p. 2318-2328.

Mallet, C. et Bretar, F., 2009. Full-Waveform Topographic Lidar: State-of-the-Art, ISPRS Journal of Photogrammetry and Remote Sensing, 64(1), p. 1-16.

Mestre, M., Hildebrand, M. et Texier, P., 2008. Un exemple d'utilisation du LiDAR sous couvert forestier guyanais, Archéopages, 23, p. 69-75.

MotкIn, D., 2001. An assessment of LIDAR for archaeological use, AARG News, 22, p. 24-25.

Pardé, J. et Bouchon, J., 1988. Dendrométrie. $2^{e}$ édition, École nationale du génie rural, des eaux et forêts, France.
Risbøl, O., GJertsen, A. K. et Skare, K., 2006. Airborne laser scanning of cultural remains in forests: some preliminary results from a Norwegian project, dans S. Campana et M. Forte (ed.), From Space to Place. $2^{\text {nd }}$ International Conference on Remote Sensing in Archaeology. Proceedings of the $2^{\text {nd }}$ International Workshop, CNR, Rome, Italy, December 4-7, 2006, p. 107-112 (BAR International Series 1568).

Sittler, B. et Hauger, K., 2007. Les apports du laser aéroporté à la documentation de parcellaires anciens fossilisés par la forêt : l'exemple des champs bombés de Rastatt en Pays de Bade, dans J.-L. Dupouey, E. Dambrine, C. Dardignac, M. Georges-Leroy (éd.). La mémoire des forêts. Actes du colloque "Forêt, Archéologie et Environnement " 14-16 décembre 2004, ONF - INRA - DRAC Lorraine, p. 155-161.

SzABó, P., 2010. Ancient woodlands boundaries in Europe, Journal of Historical Geography, 36: 205-214. 